\title{
Optimisation at the conceptual design stage with morphological indicators: design for strength or design for stiffness?
}

\author{
T. Vandenbergh ${ }^{1}$, W. P. De Wilde ${ }^{1}$ \& P. Latteur ${ }^{2}$ \\ ${ }^{I}$ Department of Mechanics of Materials and Constructions (MeMC), \\ Vrije Universiteit Brussel, Belgium \\ ${ }^{2}$ University of Gembloux, \\ Belgium and Tractebel Development Engineering, Belgium
}

\begin{abstract}
Within the framework of sustainable development we strive for constructions with a minimum volume of material. When we only consider criteria on resistance and buckling, at the stage of conceptual design a clear hierarchy among the different structural topologies can be established with Morphological Indicators (MI). MI are dimensionless numbers that represent a property of a structure (e.g. volume) and depend only on a small number of dimensionless variables (in its most simple appearance only the slenderness of the structure). We define the slenderness as $\mathrm{L} / \mathrm{H}, \mathrm{L}$ being the horizontal and $\mathrm{H}$ the vertical dimension of the rectangle that inscribes the structure. This allows one to compare the efficiency of structures objectively, with only a reduced number of variables to consider. Hence MI allow a very quick shape and topology optimisation at the conceptual design stage if only element strength (resistance) and buckling are to be considered. Though a lot of design problems, especially for lightweight structures, are characterized by stiffness constraints: global instability, upper limit on static displacements, acceptable vibrations... In such cases one of the main assumptions of the theory of MI is not valid, since fully stressed design does no longer guarantee the optimal section distribution. Therefore, problems subjected to stiffness related constraints have to be analyzed by different methods allowing the optimisation of the sizing of the different elements.

Recent research enables determination of whether one has to deal with a design for strength or a design for stiffness problems. This paper presents schematically the theory of MI and the method to distinguish a strength characterized problem from a stiffness characterized one at the conceptual design stage. An example illustrates the design process.

The principal aim is not to criticize the efficiency of the theory of MI but to point out when the theory can be applied and when not.

Keywords: conceptual design, morphological indicator, design for strength, design for stiffness, volume optimisation, fully stressed.
\end{abstract}




\section{Introduction}

Conceptual design, along with problem identification and analysis, make up the initial stage of the structural design process. Problem analysis transforms the often vague statement of a design task into a set of design requirements. Conceptual design encompasses the generation of concepts and integration into system-level solutions, leading to a relatively detailed design (Kroll et al [1]).

The assessment of structural performance is mainly, at the present time, the result of late finite element analysis processes (FEA), which remain computationally expensive, limiting their use to the analysis of a limited number of design alternatives. But, in the conceptual design stage, the quality depends on the comprehension and on the exploration of the design space (Yannou et al [2]).

During the conceptual design stage, an engineer or architect creates the general outline of a structure. In this phase a few solutions are selected out of the many possibilities because of their ability to be adapted to the most important requirements. More specifically, in the context of morphological indicators, one seeks structures with minimum volume. A good conceptual design will then yield a solution (topology and geometry) that will not (or slightly) change its relative superiority to other solutions when more detailed design calculations are performed. Hence it is very important to assess the possible impact or necessity of detailed calculations such as (global) buckling, dynamics, the weight of connections, and second order effects among others. If the context of the situation allows the use of the indicator of volume in its simplest form, a larger topological/geometrical solution space can be browsed for. This viewpoint can be expressed as follows: one should always optimise structures with a minimum number of variables as long as the detailed analysis will not alter the efficiency of the structure considerably.

\subsection{Morphological indicators}

Morphological indicators are design tools allowing the optimisation of structures (this idea was first introduced by Zalewski and Kus [3] but not named morphological indicators) at the stage of conceptual design using a limited set of parameters (Samyn [4]). The indicator of volume $W$ allows the comparison of the volume of material used for different structural systems.

$$
W=\frac{\sigma V}{F L}
$$

It is the volume of an isomorphic structure with unit span $L$, with at least one section of every element dimensioned at its unit allowable stress $\sigma$, subjected to a system of loads with unit resultant $F$.

The displacement indicator $\Delta$ compares the displacement of different structural systems.

$$
\Delta=\frac{E \delta}{\sigma L}
$$


It is the maximum displacement of an isomorphic structure with unit span $L$ in a material with unit Young's modulus $E$, with at least one section of every element dimensioned on its unit allowable stress $\sigma$, subjected to a system loads with unit resultant $F$.

The analytical expressions of both $W$ and $\Delta$ have been established by Samyn [4, 5] and Latteur [6] for trusses, beams, arches, cables, cable stayed structures, masts and frames subjected to a limited number of (simple) load cases and supports. For statically determinate structures those MI are the only function of the geometrical slenderness $L / H$ (length divided by the height of a rectangle that frames the structure). Instabilities, self weight and second order effects are neglected. Efficiency curves depicting the geometrical slenderness with respect to the minimum volume material can be established.

This figure allows the comparison between different structural typologies and topologies with a reduced number of variables. For classic truss topologies (Warren, Howe, Pratt, etc.) $W$ and $\Delta$ only depend on the number of panels and the geometrical slenderness. In a more general way, one can conclude that those MI only depend on the proportion of the rectangle in which the structure is inscribed and on the shape/topology (i.e. how this rectangle is filled). Those results are based on a fully stressed (in which every element works at its allowable stress level under at least one of the given load conditions - according to Latteur [6] groups of bars can also be used) design of statically determinate structures and for a dominant load case (in this case a uniformly distributed vertical load).

The buckling indicator $\psi$ developed by Latteur [6] takes buckling in individual elements into account.

$$
\psi=\frac{\mu \sigma L}{\sqrt{q E F}}
$$

This factor gives an indication for the buckling tendency of the compression elements in a structure with span $L$, composed of bars with a form factor $q=I / \Omega^{2}$ (Figure 1) (with $I$ the second moment of inertia and $\Omega$ the section area) in a material with Young's modulus $E$, with at least one section dimensioned on its allowable stress $\sigma$, subjected to a system of load with total resultant $F . \mu$ is the proportion of the buckling length of the compression bars over their geometrical length (which depends on the connection type, for pinned truss bars $\mu=0,9$ ).

This contribution modifies the element sizing. It implies the use of one extra parameter $(\psi)$, but increases the precision of the optimisation process. It enables the evaluation of the extra necessary volume of material to avoid buckling. Moreover, it affects the optimal solution. For example: the higher the value of the buckling indicator, the higher the optimal slenderness and number of panels for trusses. 


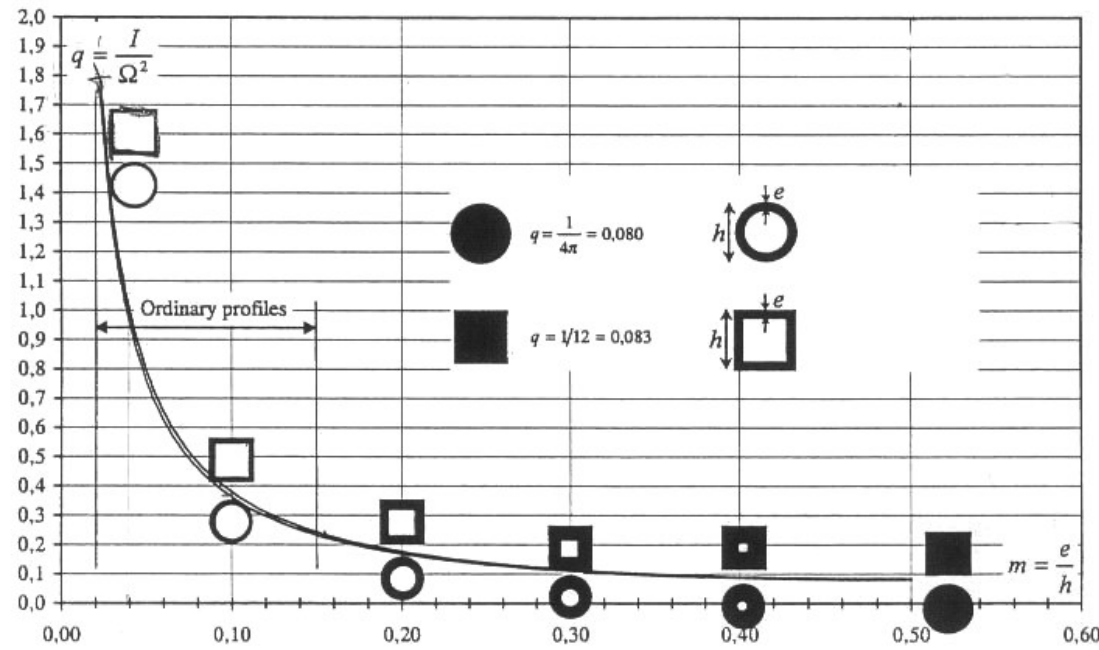

Figure 1: $\quad$ Form factor of double axis symmetric square and circular profiles [6].

The definition of the indicator of buckling clearly demonstrates that the buckling sensitivity depends on the span-load ration $(L / \sqrt{F})$. This quantity is defined as the structural index in Shanley [7]. This index illustrates that morphological indicators are dimensionless but depend upon scale effects.

\section{Problem statement}

Recent research shows that not only the volume and the maximal static displacement can be predicted at conceptual design stage, but other fundamental structural characteristics as well: Van Steirteghem [8] provides a method to evaluate the first natural eigenfrequency of a structure, introducing the indicator of fundamental eigenfrequency, which depends on the same variables as the indicator of volume and displacement. This finding is confirmed by validation of trusses in Vandenbergh et al [9].

The studies performed by Vandenbergh et al [10] and Vantomme [11] shows that the global planar stability of truss arches can be evaluated by means of the indicator of global planar stability, again depending on the same variables as the indicator of volume.

Hence it is possible to assess the strength, the stiffness and the planar stability at conceptual design stage on the basis of a limited number of variables. This essential advantage of MI can be combined with powerful search algorithms, which enables the comparison of a large number of structural morphologies, within a relatively short calculation time (Verbeeck et al $[12,13]$ and Vandenbergh et al [14]). 
Nevertheless, there is an implicit restriction on the use of the morphological indictors as optimisation tool. If all of above mentioned stiffness constraints are met, the volume indicator offers a very efficient objective function for volume minimization. However, if at least one of the stiffness constraints is violated, the designer can only modify the input variables to stiffen the structure: the slenderness, the shape and the topology. The section distribution or sizing cannot be altered since fixed by the assumption of a fully stressed design. The use of MI bases its user-friendly/efficient approach by exploring only two of the three possible optimisation branches:

- sizing: to fix the dimensions (cross section and second moment of inertia) of each element

- shape optimisation: the global form of the structure (dimensions and node configuration)

- topological optimisation: the way of connecting the different nodes

The use of MI considers implicitly fully stressed design as being the optimal sizing and therefore does not further explore the first branch. The optimisation process is carried out on the shape and topology. In case only strength and element buckling are the dimensioning design criteria, this is a correct working method. When one of the stiffness constraints is not met, this assumption is no longer valid. The use of fully stressed design does not allow an alternative sizing and can therefore lead to unacceptable results.

\section{Example of applicability limits}

The 2D-example shown in Fig. 2 illustrates the limit of optimisation with MI. Assume a statically determinate Warren truss of $S 235$-steel, charged by a uniformly distributed load on the lower truss chord with total value $F=800 k N$, from which $60 \%$ can be considered to be covibrating. The shape and topology are fixed by a slenderness $L / H=10$ and 8 panels (this leads to low angles between the different elements (here $\alpha=38.6^{\circ}$ and $\beta=102.8^{\circ}$ in Fig. 3), though these exceed the values given by the European design standards [15] which proscribe minimal angles of $30^{\circ}$ for direct connections between tubular elements). Tubular sections are chosen with thickness to diameter ratio of $3.8 \%$ (resulting in $q=1$, see Fig. 1). These values allow calculating the indicator of buckling

$$
\psi=\frac{\mu \sigma L}{\sqrt{q E F}}=\frac{0,9 \cdot 235 M P a \cdot 20 m}{\sqrt{1.210 G P a .800 k N}}=10,32
$$

The indicator of volume and displacement can be evaluated graphically or numerically with Latteur [6]

$$
W=\frac{\sigma V}{F L}=2,42
$$




$$
\Delta=\frac{\delta E}{\sigma L}=3,32
$$

As mentioned in the introduction, these values respectively give an indication for the volume and the maximal displacement of the fully stressed Warren truss, considering buckling for the compressed bars.

The total covibrating load is the sum of the external covibrating load and the selfweight. Hence, the ratio of total covibrating load in SLS over the external load in ULS (Serviceability Limit State and Ultimate Limit State, as defined in the European Standards [13]) is

$$
z^{*}=y^{*}+\frac{\rho \cdot L \cdot W}{1,35 \cdot \sigma}=60 \%+\frac{77009 N / m^{3} \cdot 20 m \cdot 2,42}{1,35 \cdot 235 M P a}=61,2 \%
$$

According to Van Steirteghem [8] and Vandenbergh et al [9], the fundamental eigenfrequency of the fully stressed truss can be predicted using the indicator of displacement

$$
f_{\text {eig }}=\frac{c_{c o r}}{2 \pi} \sqrt{\frac{9.81 m / s^{2} \cdot E}{\Delta \sigma L z^{*}}}=\frac{1,14}{2 \pi} \sqrt{\frac{9.81 \mathrm{~m} / \mathrm{s}^{2} \cdot 210 \mathrm{GPa}}{3,32.235 \mathrm{MPa} \cdot 20 \mathrm{~m} \cdot 0.612}}=2,66 \mathrm{~Hz}
$$

with

$c_{c o r}$ a correction factor due to the use of a SDOF-model value for the calculation of the fundamental eigenfrequency, for trusses 1,14 (Van Steirteghem [8])

One supposes that a minimal eigenfrequency of $3 \mathrm{~Hz}$ is required in order to avoid resonance phenomena. The shape and topology are kept unmodified. First, a global stress level reduction is introduced to increase the truss stiffness and hereby the fundamental eigenfrequency. The allowable stress level is decreased by a factor $\beta<1$. This global stress level reduction does not affect the relative section distribution, since it increases every bar section with the same proportionality (Figure 2, upper). Therefore no additional input parameters are necessary. This solution yields a stress level reduction of $\beta=43,3 \%$, a volume indicator $W=3,75$ and a displacement indicator $\Delta=2,59$.

Another possibility is to use the 'first optimisation branch' mentioned above: sizing. Every section can be selected freely with a minimum value imposed by the resistance and/or bar buckling criteria. The disadvantage is that the concept of morphological indicator is not longer necessary, since no fully stressed design is used. The advantage is a much wider search space for possible solutions, resulting in a lighter truss. Here the optimal section distribution is illustrated in Figure 2 (lower). This solution yields a stress level reduction of $\beta=100 \%$, a volume indicator $W=3,01$ and a displacement indicator $\Delta=2.61$. 

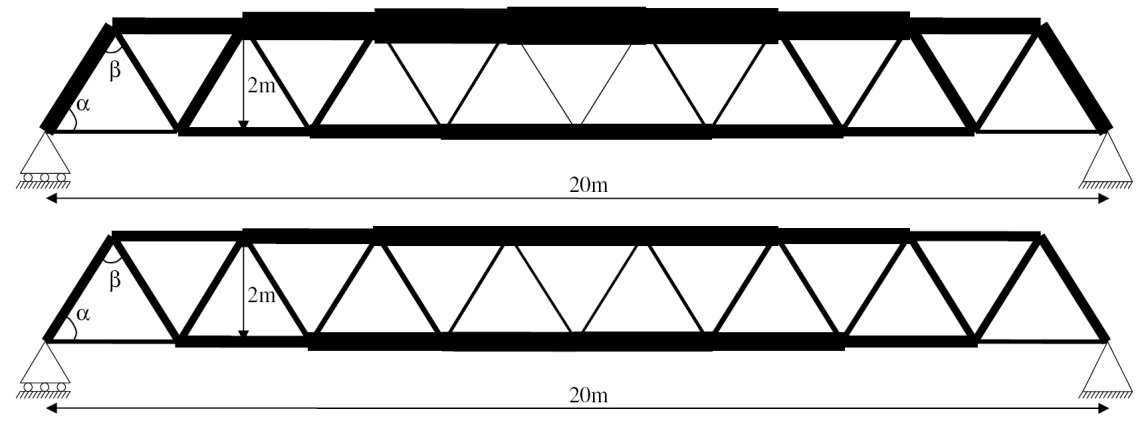

Figure 2: $\quad$ Statically determined Warren truss under uniformly distributed load on lower chord. Section distribution for fully stressed with global stress level reduction (upper) and optimal section distribution (lower).

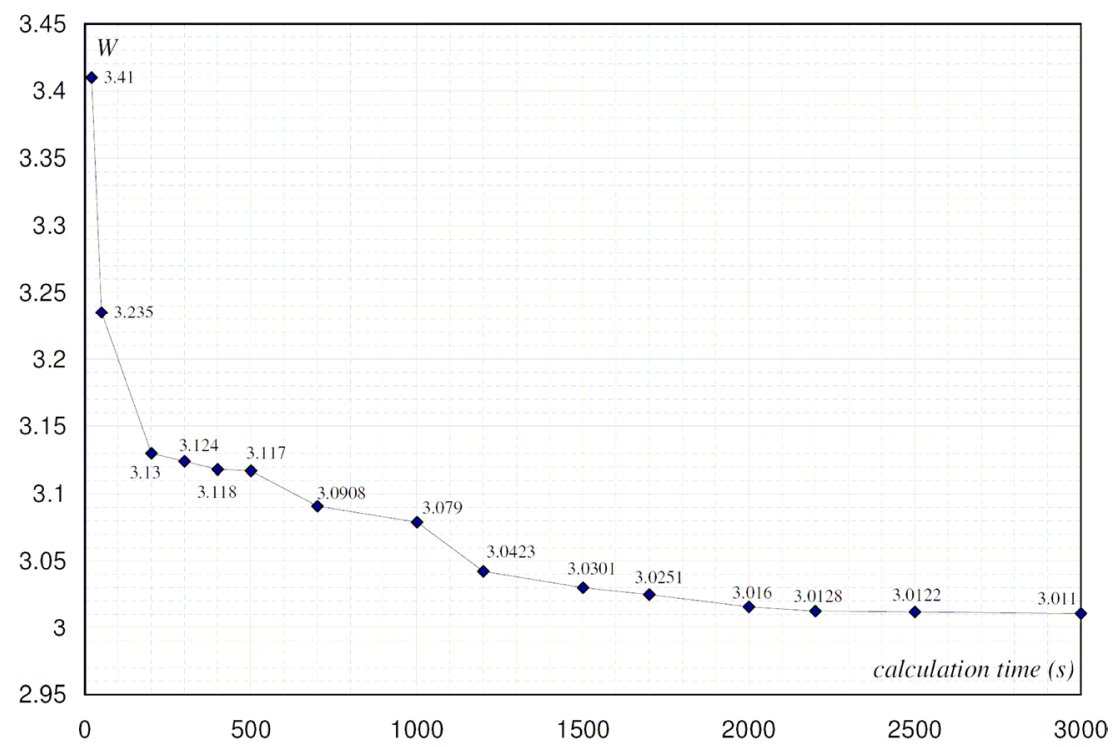

Figure 3: Minimal volume indicator for free sizing in function of the GA calculation time.

The corresponding volume gain compared to the fully stressed solution with global stress level reduction is $19.7 \%$.

Finally, it should be mentioned that allowing free sizing increases the number of variables considerably (one extra variable for every additional free section size). Hence, the calculation time increases. In this paper a genetic algorithm is used to find the optimal section distribution. Figure 3 illustrates the convergence of the minimal volume indicator as a function of the calculation time. 
This example illustrates that fully stressed design does not always represent the best/lightest sizing method, if other design constraints are considered (here a lower limit on the fundamental eigenfrequency). This conclusion is also valid for other design criteria (static displacements, global instability [16], etc.) and other structures (arches, trusses, etc.).

\section{Conclusion}

In structural optimisation one can state that if at least one of the stiffness constraints (acceptable static displacements, global stability and absence of resonance) is not met, fully stressed design does not guarantee the most efficient solution. Hence, up to now, morphological indicators are used to offer a very performing conceptual optimisation tool in a design for strength approach. In design for stiffness one needs different optimisation techniques and/or to accept that every additional non-fully stressed section stands for an additional input variable to optimise. Analysis of the indicator of displacement, the indicator of first eigenfrequency and the indicator of global planar stability prove that even at the stage of conceptual design one can determine whether design for stiffness or design for strength is the designing parameter (Figure 4).

To summarize, one can state that the power of the morphological indicators, which are dependent on a limited number of design variables, follows from the fixed sizing. However, the downside of the medal is that sizing represents one of the fundamental optimisation branches, which should be used in design for stiffness problems. Though, the evaluation of the morphological indicators discussed in this paper enables to determine whether one has to deal with such a design for stiffness problem or not.

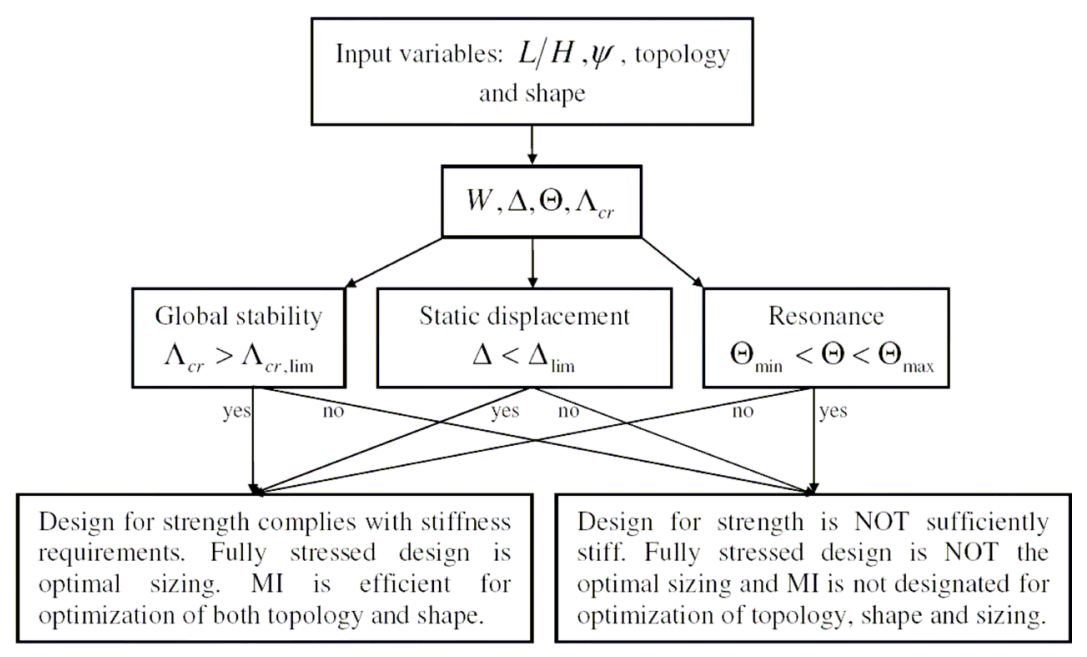

Figure 4: $\quad$ Flowchart for the use of morphological indicators at conceptual design stage. 


\section{Acknowledgements}

The authors want to thank the Institute for the Promotion of Innovation through Science and Technology in Flanders (IWT Vlaanderen), which funds this research.

\section{References}

[1] Kroll, E., Condoor, S. \& Jansson, D. G., Innovative Conceptual Design Theory and Application of Parameter Analysis, Cambridge University Press: Great Britain, 2001.

[2] Yannou, B., Hamdi, A. \& Landel, E., Une stratégie de modélisation conceptuelle pour la prise en compte de performances vibro-acoustiques en préconception d'un berceau automobile, Mécanique \& industries (Méc. ind.), Elsevier: France, 2000.

[3] Zalewski, W. \& Kus, S., Shaping structures of least-weight, Proceedings of the International Conference on Lightweight Structures in Civil Engineering, ed. J.B. Obrebski, Magat-Magdalena Burska: Warsaw, Poland, pp. 153-157, 1995.

[4] Samyn, P., Etude Comparée du Volume et du déplacement de Structures Isostatiques Bidimensionnelles sous Charges Verticales entre Deux Appuis. Vers un outil d'évaluation de prédimensionnement des structures (Tome 1 à 4), PhD Thesis, Université de Liège: Belgium, 1999.

[5] Samyn, P., Etude de la morphologie des structures à l'aide des indicateurs de volume et de déplacement, Académie Royale de Belgique, Classes des Sciences: Belgium, 2004.

[6] Latteur, P., Optimisation et Prédimensionnement des Treillis, Arcs, Poutres et Câbles sur Base d'Indicateurs Morphologiques (Tome 1 à 4), PhD Thesis, Vrije Universiteit Brussel: Belgium, 2000.

[7] Shanley, FR., Weight-strength analysis of aircraft structures, Dover Publications, Inc: New York, 1960.

[8] Van Steirteghem, J., A Contribution to the Optimisation of Structures Using Morphological Indicators: (In)Stability and Dynamics, PhD Thesis, Vrije Universiteit Brussel: Belgium, 2005.

[9] Vandenbergh, T., Verbeeck, B. \& De Wilde, W. P., Dynamical analysis and optimisation of statically determinate trusses at conceptual design stage, Proceedings for the ECCOMAS Thematic Conference (COMPDYN 2007) on Computational Methods in Structural Dynamics and Earthquake Engineering, eds. M. Papadrakakis, D.C. Charmpis, N.D. Lagaros \& Y. Tsompanakis, p253, 2007.

[10] Vandenbergh, T., De Wilde, W. P., Latteur, P. \& Vantomme, L., Optimisation of Statically Determinate Trusses Considering Planar Instabilities at Conceptual Design Stage, Proceedings for the Third International Congress dedicated to the "art, science and practice of structural engineering”, p. 141, 2007. 
[11] Vantomme, L., Uitbreiding van de theorie van de morfologische indicatoren naar het specifiek geval van de parabolische vakwerkboog: ontwerprichtlijnen en -strategie, Master Thesis, Vrije Universiteit Brussel: Belgium, 2007.

[12] Verbeeck, B., De Wilde, W. P., Samyn, P. \& Van Steirteghem, J., The Use of Genetic Algorithms and Morphological Indicators in the Optimisation of 2D Trusses, Proceedings for the First International Conference on High Performance Structures and Materials, eds. C.A. Brebbia, W.P. De Wilde, WIT Press: Southampton, UK, pp. 571-577, 2004.

[13] Verbeeck, B., De Wilde, W.P., Samyn, P. \& Van Steirteghem, J., The Need of Numerical Techniques for the Optimisation of Structures Using Morphological Indicators, Proceedings for the Second International Conference on High Performance Structures and Materials, eds. S. Hernandez, C.A. Brebbia, WIT Press: Southampton, UK, pp. 65-72, 2005.

[14] Vandenbergh, T., Verbeeck, B., De Wilde, W. P. \& Latteur, P., The Use of Morphological Indicators and Genetic Algorithms in Structural Optimisation Considering Stiffness Constraints, Proceedings of The Eighth International Conference on Computational Structures Technology, eds. B. H. V. Topping, G. Montero \& R. Montenegro, Civil-Comp Press: Stirlingshire, United Kingdom, paper 201, 2006.

[15] Eurocode - Design of steel structures Part 1-8: Design of joints. European Standard NBN EN 1993-1-8, (7.1.2) p 101, 2005.

[16] Vandenbergh, T., De Wilde, W.P., Optimisation at conceptual design stage: pros and cons, (submitted in Advances in Engineering Software), 2008. 